\title{
Pharmacovigilance of patients with multiple myeloma being treated with bortezomib and/or thalidomide
}

\author{
T.B.M. Castro ${ }^{1}$, A.E. Hallack Neto $^{2,3}$, A. Atalla ${ }^{3}$ and L.C. Ribeiro ${ }^{4}$ \\ ${ }^{1}$ Programa de Pós Graduação em Saúde Brasileira, Faculdade de Medicina, Universidade Federal de Juiz de Fora, \\ Juiz de Fora, MG, Brasil \\ ${ }^{2}$ Departamento de Clínica Médica, Faculdade de Medicina, Universidade Federal de Juiz de Fora, Juiz de Fora, MG, Brasil \\ ${ }^{3}$ Serviço de Hematologia e Transplante de Medula Óssea do Hospital Universitário, Faculdade de Medicina, \\ Universidade Federal de Juiz de Fora, Juiz de Fora, MG, Brasil \\ ${ }^{4}$ Departamento de Estatística, Universidade Federal de Juiz de Fora, Juiz de Fora, MG, Brasil
}

\begin{abstract}
In order to evaluate the main adverse effects of drug protocols using bortezomib and/or thalidomide for the treatment of multiple myeloma, we conducted a prospective study. Data were collected through interviews, clinical observation, and from hospital records. A total of 59 patients were included. There was a predominance of females, $36(61 \%)$ vs $23(39 \%)$ males, and of whites, $49(83.1 \%)$ vs $10(16.9 \%)$ blacks. Age ranged from 40 to 94 years, with a median of 65 years (SD=11.6). Regarding staging at diagnosis, $27(45.7 \%)$ patients were in stage III-A, with $12(20.3 \%)$ patients having serum creatinine $\geqslant 2 \mathrm{mg} / \mathrm{dL}$. The main adverse effects in the bortezomib treatment group $(n=40)$ were: neutropenia $(42.5 \%)$, diarrhea $(47.5 \%)$, and peripheral neuropathy in $60 \%$ of cases, with no difference between the $i v(n=26)$ and $s c(n=14)$ administration routes $(P=0.343)$. In the group treated with thalidomide $(n=19), 31.6 \%$ had neutropenia, $47.4 \%$ constipation, and $68.4 \%$ peripheral neuropathy. Neutropenia was associated with the use of alkylating agents $(P=0.038)$. Of the 3 patients who received bortezomib in combination with thalidomide, only 1 presented peripheral neuropathy (33.3\%). Peripheral neuropathy was the main adverse effect of the protocols that used bortezomib or thalidomide, with a higher risk of neutropenia in those using alkylating agents. Improving the identification of adverse effects is critical in multiple myeloma patient care, as the patient shows improvements during treatment, and requires a rational and safe use of medicines.
\end{abstract}

Key words: Multiple myeloma; Side effects; Bortezomib; Thalidomide

\section{Introduction}

Multiple myeloma (MM) is a neoplasm with an unclear etiology, having an incidence of about 4 per 100,000, and accounting for $1 \%$ of all malignancies and $10 \%$ of hematologic malignancies (1). Treatment of the disease consists of chemotherapy, antiangiogenic agents, immunomodulators, and autologous stem cell transplants (2). Noteworthy, among the drugs that comprise the therapeutic arsenal for MM treatment are immunomodulators and proteasome inhibitors (3).

Most of the treatment protocols used in Brazil for treating $\mathrm{MM}$ are composed of bortezomib and/or thalidomide, combined with other drugs such as alkylating agents and corticosteroids (1). Bortezomib is a proteasome inhibitor and thalidomide is an immunomodulatory drug, and both have mechanisms of action that are not yet fully understood $(4,5)$.

During chemotherapy, patients are highly likely to present some sort of adverse effect. Therefore, the attempt to understand the mechanisms involved in these effects, the extent of the risk, and the strategies to control them is part of an adequate patient care that the health care team should provide to patients $(1,6,7)$. In this context, pharmacovigilance, which is an activity carried out by the professional pharmacist and is related to the detection, understanding, assessment, and prevention of adverse reactions, proves to be an important tool during chemotherapy (8). Through this method, we aimed to assess the adverse effects of drug protocols using bortezomib and/or thalidomide in patients with $\mathrm{MM}$, as well as to grade and identify the main adverse reactions.

\section{Material and Methods}

\section{Study design}

This was a multicenter, prospective, observational study, approved by the Ethics Committee of the Hospital Universitário, Universidade Federal de Juiz de Fora. 
Table 1. Peripheral neuropathy grading criteria according to the National Cancer Institute, version 4.0.

\begin{tabular}{lc}
\hline Grade & Symptoms \\
\hline 1 & Asymptomatic: Loss of deep tendon reflexes or paresthesia \\
2 & Moderate symptoms: Limitations on instrumental activities of daily living \\
3 & Severe symptoms: Limitations on self-care activities \\
4 & Life-threatening consequences: urgent intervention indicated \\
5 & Death \\
\hline
\end{tabular}

The informed consent form was signed by the researcher and patients.

The inclusion criteria for the study were: patients with MM; adults over 18 years old; elective and non-elective for bone marrow transplant and being under treatment protocols using bortezomib and/or thalidomide. There were no restrictions regarding the presence of comorbidities and use of concomitant medications.

The pharmacovigilance strategy included questionnaire-based interviews with the patient at each appointment, clinical observation, and consultation of laboratory tests and medical records.

The interviews were conducted according to the type of medication used by the patient. For example: patients that were taking bortezomib, or bortezomib and thalidomide combination were interviewed at the beginning of each cycle, and data analysis accounted for the cycle they were in; patients using only thalidomide were interviewed once a month (on the day of bisphosphonate infusion) and data analysis accounted for the number of months on medication, relating the findings to this temporal quantification.

\section{Toxicity monitoring}

The studied adverse effects were those most frequently described in the literature and in the bortezomib and thalidomide package inserts, specifically blood and lymphatic system, gastrointestinal, and nervous system disorders. The main blood and lymphatic system disorders studied were anemia, neutropenia, and thrombocytopenia. Anemia was considered only in patients with diminished hemoglobin levels throughout the treatment. The other disorders evaluated were constipation and diarrhea, and peripheral neuropathy (PN). Blood, lymphatic system, and gastrointestinal disorders were not graded. However, PN was graded according to the National Cancer Institute $(\mathrm{NCl})$ criteria, version 4.0, as shown in Table 1.

Data were collected at the University Hospital of the Federal University of Juiz de Fora, the Maria José Baeta Reis Hospital (Ascomcer), and the Radiotherapy and Nuclear Medicine Center, Ltd. (Centerq), from May 2013 to August 2015.

\section{Statistical analysis}

For statistical analysis and database management, SPSS (Statistical Package for the Social Sciences, IBM,
USA) software was used. Descriptive analysis of categorical variables was performed using frequencies, means, medians, and standard deviations. The evaluation of toxicity and tolerance was carried out using Fisher's exact test or chisquare $(\chi 2)$ test for heterogeneity and for trend. A $5 \%$ significance level was adopted.

\section{Results}

A total of 59 patients were included in the study (Table 2). As the chemotherapy protocol of 3 patients was

Table 2. Profile of the multiple myeloma patients under treatment with bortezomib, thalidomide, or a combination of both drugs.

\begin{tabular}{lcr}
\hline Profile of patients & $\mathrm{n}=59$ & $\%$ \\
\hline Age (years) & $65(40-94)$ & - \\
Gender & & \\
$\quad$ Male & 23 & 39.0 \\
Female & 36 & 61.0 \\
Immunoglobulin & & \\
$\quad$ IgG & 34 & 57.5 \\
IgA & 14 & 23.8 \\
Light chain & 6 & 10.2 \\
$\quad$ Data not determined & 5 & 8.5 \\
Race & & \\
White & 49 & 83.1 \\
$\quad$ Black & 10 & 16.9 \\
Relapsed & 17 & 28.8 \\
ASCT & 5 & 8.5 \\
Comorbidities & 31 & 52.5 \\
Allergy & 8 & 13.6 \\
Use of natural products & 18 & 30.5 \\
Alcoholic & 12 & 20.3 \\
Smoker & 4 & 6.8 \\
Polypharmacy & 40 & 67.8 \\
Performance status & & \\
90-100\% & 19 & 32.2 \\
70-80\% & 29 & 49.2 \\
$50-60 \%$ & 3 & 13.5 \\
30-40\% & & 5.1 \\
\hline & &
\end{tabular}

Age is reported as median (range). IgG: immunoglobulin G; IgA: immunoglobulin A; ASCT: autologous stem cell transplants. 
Table 3. Durin-Salmon staging and risk stratification of multiple myeloma patients under treatment with bortezomib, thalidomide, or a combination of both drugs.

\begin{tabular}{lrr}
\hline Staging and risk stratification & $\mathrm{n}=59$ & $\%$ \\
\hline II-A & 9 & 15.3 \\
III-A & 27 & 45.7 \\
II-B & 2 & 3.4 \\
III-B & 10 & 16.9 \\
Data not determined & 11 & 18.7 \\
\hline
\end{tabular}

Data are reported as number $(\mathrm{n})$ and percentage (\%). A: serum creatinine $<2 \mathrm{mg} / \mathrm{dL}$; $B$ : creatinine $\geqslant 2 \mathrm{mg} / \mathrm{dL}$.

modified during the study, 62 treatments were evaluated. Bortezomib was used in 40 patients, being administered intravenously (iv) in 26 and subcutaneously (sc) in 14. Bortezomib was combined with alkylating agents in 36 treatments. Thalidomide was used in 19 patients, 12 of them taking it in combination with alkylating agents. Protocols with bortezomib and thalidomide were used by 3 patients, and in combination with alkylating agents in 3 cases.

For 5 patients $(8.5 \%)$, the type of immunoglobulin secreted was not determined. The most frequently found immunoglobulin was $\operatorname{lgG}$, found in 34 (57.5\%) patients, followed by $\operatorname{lgA}$, found in $14(23.8 \%)$, and light chain, found in $6(10.2 \%)$.

In Table 3, we show the Durie-Salmon MM classification for the study participants, which accounts for disease stage (I, II, III) and risk (A, serum creatinine $<2 \mathrm{mg} / \mathrm{dL}$; and $B$, creatinine $\geqslant 2 \mathrm{mg} / \mathrm{dL}$ ). Twenty-seven $(45.7 \%)$ patients were in stage III-A, showing a poor prognosis and advanced illness. There were $12(20.3 \%)$ patients with creatinine $\geqslant 2 \mathrm{mg} / \mathrm{dL}$.
The main adverse reactions observed in patients during the study are reported in Table 4. Nervous system disorders were observed in the 3 groups of patients. PN was present in $24(60 \%)$ patients among those treated with bortezomib protocols (without thalidomide), in 13 $(68.4 \%)$ patients among those treated with thalidomide, and in $1(33.3 \%)$ patient treated with the bortezomib + thalidomide protocol. Gastrointestinal disorders (diarrhea and/or constipation) were observed in 30 of the 40 patients treated with bortezomib protocols, with diarrhea being slightly more frequent $(47.5 \%)$ than constipation $(42.5 \%)$. Among the 19 patients treated with thalidomide, $13(68.4 \%)$ had PN, 3 of them with $\geqslant 2$ grade. Constipation was observed in $47.4 \%$ of patients treated with thalidomide, and was unrelated to the exposure time of the medication $(P=0.370)$. No episodes of diarrhea were reported. Patients treated with the combination of thalidomide and bortezomib presented no gastrointestinal disorders, which may be explained by the small number of patients in this category $(n=3)$. Table 5 shows the frequency of blood and lymphatic system disorders between patients taking or not alkylating agents, and Table 6 shows the frequency of PN between patients to whom bortezomib was administered iv or sc, excluding patients who were treated with combined bortezomib and thalidomide protocols. Two patients treated with bortezomib protocols administered intravenously showed a PN grade $\geqslant 2$. In one case, there was a need for dose adjustment, and carbamazepine was indicated.

The nervous system and gastrointestinal disorders were compared between patient treatment time. The patients treated with bortezomib protocols were separated into a group with up to 4 treatment cycles vs a group with more than 4 treatment cycles. Meanwhile, patients submitted to thalidomide protocols were separated into a group treated for up to 4 months vs a group treated for

Table 4. Main adverse reactions in multiple myeloma patients, according to their treatment protocol.

\begin{tabular}{|c|c|c|c|c|c|c|}
\hline \multirow[t]{2}{*}{ Adverse reactions } & \multicolumn{2}{|c|}{$\begin{array}{l}\text { Bortezomib } \\
\qquad(\mathrm{N}=40)\end{array}$} & \multicolumn{2}{|c|}{$\begin{array}{l}\text { Thalidomide } \\
\qquad(\mathrm{N}=19)\end{array}$} & \multicolumn{2}{|c|}{$\begin{array}{c}\text { Bortezomib }+ \\
\text { Thalidomide }(\mathrm{N}=3)\end{array}$} \\
\hline & $\mathrm{n}$ & $\%$ & $\mathrm{n}$ & $\%$ & $\mathrm{n}$ & $\%$ \\
\hline \multicolumn{7}{|c|}{ Blood and lymphatic system disorders } \\
\hline Anemia & 15 & 37.5 & 3 & 15.8 & 2 & 66.7 \\
\hline Neutropenia & 17 & 42.5 & 6 & 31.6 & 2 & 66.7 \\
\hline Thrombocytopenia & 13 & 32.5 & 3 & 15.8 & 3 & 100.0 \\
\hline \multicolumn{7}{|l|}{ Gastrointestinal disorders } \\
\hline Constipation & 17 & 42.5 & 9 & 47.4 & 0 & 0.0 \\
\hline Diarrhea & 19 & 47.5 & 0 & 0.0 & 0 & 0.0 \\
\hline \multicolumn{7}{|l|}{ Nervous system disorders } \\
\hline Peripheral neuropathy & 24 & 60.0 & 13 & 68.4 & 1 & 33.3 \\
\hline
\end{tabular}

Data are reported as number and percentage (\%). 
Table 5. Frequency of blood and lymphatic system disorders, according to the presence or not of alkylating agents in the treatment protocol in multiple myeloma patients under treatment with bortezomib, thalidomide, or a combination of both drugs.

\begin{tabular}{|c|c|c|c|c|c|c|}
\hline \multirow[t]{2}{*}{$\begin{array}{l}\text { Blood and lymphatic } \\
\text { system disorders }\end{array}$} & \multicolumn{2}{|c|}{$\begin{array}{l}\text { With alkylating } \\
\text { agents }(\mathrm{N}=51)\end{array}$} & \multicolumn{2}{|c|}{$\begin{array}{l}\text { Without alkylating } \\
\text { agents }(\mathrm{N}=11)\end{array}$} & \multirow[t]{2}{*}{$\mathrm{P}$} & \multirow[t]{2}{*}{ OR } \\
\hline & $\mathrm{n}$ & $\%$ & $\mathrm{n}$ & $\%$ & & \\
\hline Anemia & 18 & 35.3 & 2 & 18.2 & 0.478 & 2.45 \\
\hline Neutropenia & 24 & 47.1 & 1 & 9.1 & 0.038 & 8.88 \\
\hline Thrombocytopenia & 14 & 33.3 & 2 & 18.2 & 0.478 & 2.25 \\
\hline
\end{tabular}

Data are reported as number and percentage (\%). Statistical analysis was carried out with the chi-square test. OR: odds ratio.

Table 6. Frequency of peripheral neuropathy according to the route of administration of bortezomib in multiple myeloma patients.

\begin{tabular}{|c|c|c|c|c|c|c|}
\hline \multirow[t]{2}{*}{ Nervous system disorder } & \multicolumn{2}{|c|}{$\begin{array}{l}\text { Intravenous } \\
(\mathrm{N}=26)\end{array}$} & \multicolumn{2}{|c|}{$\begin{array}{l}\text { Subcutaneous } \\
\qquad(N=14)\end{array}$} & \multirow[t]{2}{*}{$\mathrm{P}$} & \multirow[t]{2}{*}{ OR } \\
\hline & $\mathrm{n}$ & $\%$ & $\mathrm{n}$ & $\%$ & & \\
\hline Peripheral neuropathy grade 1 & 15 & 57.7 & 7 & 50 & 0.641 & 1.36 \\
\hline Peripheral neuropathy grade $\geqslant 2$ & 2 & 7.7 & 0 & 0 & 0.533 & - \\
\hline Total & 17 & 65.4 & 7 & 50 & 0.343 & 1.89 \\
\hline
\end{tabular}

Data are reported as number and percentage (\%). Statistical analysis was carried out with the chi-square test. OR: odds ratio.

more than 4 months (Table 7). The time of exposure to bortezomib and the development of nervous system disorders did not show an association $(P=0.505)$. Patients treated with combined bortezomib and thalidomide protocols were excluded from this analysis, which was conducted independently of the bortezomib administration route.

\section{Discussion}

In this study, there was a predominance of females compared to males. These data differ from those described in the literature, where a predominance of males in $\mathrm{MM}$ is observed $(9,10)$. This inconsistency may be explained by our relatively small sample size and/or specific characteristics of the study population.

The majority of the participants in our study were white, which contrasts with the literature, where a higher incidence of MM in blacks is reported $(11,12)$. However, other studies $(9,13)$ have reported a predominance of whites; for example, the study by Hungria et al. (13) reported a high prevalence of white/Caucasian (83.3\%) in MM patients from 16 Brazilian institutions.

Patient median age of 65 years was very similar to other studies conducted in Brazil, with mean ages at diagnosis of 66 and 60.5 years $(9,13)$. A number of studies show that the elderly are more likely to experience adverse drug reactions, which can be explained by the physiological changes that come with aging, as well as by alterations in the pharmacokinetics and pharmacodynamics of the body (14-16).

The results concerning immunoglobulins were similar to those found in a study by Kyle et al. (9). Our findings concerning disease stage were similar to a study conducted in Brazil in 2008 (13) with 1,112 patients showing that the majority of participants were already in an advanced stage of the disease at the time of diagnosis.

The most frequently found adverse reactions were blood and lymphatic system, gastrointestinal, and nervous system disorders, which are commonly observed in patients treated with chemotherapy protocols using bortezomib and/or thalidomide (17-19). Blood and lymphatic system disorders, such as leukopenia, neutropenia, and thrombocytopenia, are associated mainly with the use of alkylating agents, which act in tissues that have rapid proliferation, a high mitotic index, and a short cell cycle (17). In our study, neutropenia was significantly associated with the use of alkylating agents, however anemia and thrombocytopenia were not.

Nervous system disorders were observed in the 3 groups of patients in our study. A prospective study 
Table 7. Main observed adverse effects according to exposure time to bortezomib or thalidomide in multiple myeloma patients.

\begin{tabular}{|c|c|c|c|c|c|c|c|c|c|c|}
\hline \multirow[t]{3}{*}{ Main adverse effects } & \multicolumn{4}{|c|}{ Bortezomib } & \multirow{3}{*}{$\mathrm{P}$} & \multicolumn{4}{|c|}{ Thalidomide } & \multirow{3}{*}{$\mathrm{P}$} \\
\hline & \multicolumn{2}{|c|}{$\begin{array}{c}\leqslant 4 \text { cycles } \\
(\mathrm{N}=15)\end{array}$} & \multicolumn{2}{|c|}{$\begin{array}{c}>4 \text { cycles } \\
(\mathrm{N}=25)\end{array}$} & & \multicolumn{2}{|c|}{$\begin{array}{c}\leqslant 4 \text { months } \\
(\mathrm{N}=10)\end{array}$} & \multicolumn{2}{|c|}{$\begin{array}{c}>4 \text { months } \\
(\mathrm{N}=9)\end{array}$} & \\
\hline & $\mathrm{n}$ & $\%$ & $\mathrm{n}$ & $\%$ & & $\mathrm{n}$ & $\%$ & $\mathrm{n}$ & $\%$ & \\
\hline Constipation and/or diarrhea & 9 & 60.0 & 21 & 84 & 0.135 & 6 & 60 & 3 & 33.3 & 0.370 \\
\hline Peripheral neuropathy & 8 & 53.3 & 16 & 64 & 0.505 & 7 & 70 & 6 & 66.7 & 1.000 \\
\hline
\end{tabular}

Data are reported as number and percentage (\%). $\mathrm{N}$ corresponds to the number of treatments in each group according to exposure to bortezomib or thalidomide; $\mathrm{n}$ corresponds to the number of gastrointestinal and nervous system disorders that occurred. Statistical analysis was carried out with Fisher's exact test.

by Richardson et al. (19) analyzed the efficacy and safety of bortezomib for the treatment of $\mathrm{MM}$, the presence of PN as a standard adverse effect, and the genetic pre-disposition of patients for the development of neuropathies. Of the 64 patients studied, 41 presented PN.

The subcutaneous (sc) administration of bortezomib has proven to be more efficacious compared to $i v$, in addition to reducing adverse effects, such as PN (20). Although the difference between $s c$ and iv administration was not significant, studies show that there is a decrease of $\mathrm{PN}$ when bortezomib is administered sc, especially higher grade PN $(20,21)$.

Gastrointestinal disorders are common side effects in patients using bortezomib (19). Similarly, in our study, gastrointestinal disorders (diarrhea and/or constipation) were observed in $75 \%$ of the patients treated with bortezomib protocols. The pathogenesis of diarrhea caused by bortezomib is not yet clear. It was believed that this proteasome inhibitor did not cause mucosal damage, but a recent report has described mucositis in the colon of patients under bortezomib treatment (22).

Mileshkin et al. (23) conducted a study to evaluate PN in $\mathrm{MM}$ patients being treated with thalidomide. Among 75 patients, $41 \%$ developed PN and 15\% discontinued treatment due to this toxicity. The relationship between the exposure time to thalidomide and $\mathrm{PN}$ is still not fully clarified (23). Our findings showed that the time of

\section{References}

1. Almeida JRC. Farmacêuticos em oncologia: uma nova realidade. 2nd edn. São Paulo: Atheneu; 2010.

2. Kumar SK, Rajkumar SV, Dispenzieri A, Lacy $M Q$, Hayman SR, Buadi FK, et al. Improved survival in multiple myeloma and the impact of novel therapies. Blood 2008; 111: 2516-2520, doi: 10.1182/blood-2007-10116129

3. Morgan GJ, Kaiser MF. How to use new biology to guide therapy in multiple myeloma. Hematology Am Soc Hematol exposure to thalidomide and $\mathrm{PN}$ was not significantly associated. Constipation was a common adverse reaction in patients treated with thalidomide, which is in accordance to other studies. It has been hypothesized that this gastrointestinal disorder is a side effect of thalidomide action on the nerve endings of the intestine, as occurs with other neurotoxic agents such as vincristine (18).

Our overall findings were similar to those from Brazilian and international literature $(13,19,23)$, showing a high frequency of side effects. Although we have not assessed the impact on quality of life of our patients, we infer that such events have a negative impact, leading us to believe that the improvement in the identification of adverse events is extremely important for the care of the MM patient. Since the majority of the adverse drug reactions are nonspecific, they may very often be confused with symptoms of the disease or from other causes. Studies on pharmacovigilance should be encouraged in order to achieve a better understanding of these adverse effects, which will allow for a greater adherence to treatment and consequently improve prognosis and quality of life for these patients.

\section{Acknowledgments}

We wish to thank the patients who participated in this study. We would also like to thank the professionals and institutions that collaborated in this research.

Educ Program 2012; 2012: 342-3491, doi: 10.1182/asheducation-2012.1.342

4. Vacca A, Scavelli C, Montefusco V, Di Pietro G, Neri A, Mattioli $M$, et al. Thalidomide downregulates angiogenic genes in bone marrow endothelial cells of patients with active multiple myeloma. J Clin Oncol 2005; 23: 5334-5346, doi: 10.1200/JCO.2005.03.723

5. Painuly U, Kumar S. Efficacy of bortezomib as first-line treatment for patients with multiple myeloma. Clin 
Med Insights Oncol 2013; 7: 53-73, doi: 10.4137/CMO. S7764

6. Shimada CS. Efeitos adversos no tratamento quimioterápico: uma visão para enfermeiros e farmacêuticos. São Paulo: Planmark; 2009.

7. Saad ED, Hofff PM, Carnelós RP, Katz A, Novis YAS, Pietrocola $\mathrm{M}$, et al. Critérios comuns de toxicidade do Instituto Nacional de Câncer dos Estados Unidos. Rev Bras Cancerol 2002; 48: 63-96.

8. Varallo F, Mastroianni PC. Farmacovigilância: da teoria à prática. São Paulo: Unesp; 2013.

9. Kyle RA, Gertz MA, Witzig TE, Lust JA, Lacy MQ, Dispenzieri A, et al. Review of 1027 patients with newly diagnosed multiple myeloma. Mayo Clin Proc 2003; 78: 21-33, doi: 10.4065/78.1.21

10. Palumbo A, Avet-Loiseau $H$, Oliva $S$, Lokhorst HM, Goldschmidt H, Rosinol L, et al. Revised international staging system for multiple myeloma: A report from International Myeloma Working Group. J Clin Oncol 2015; 33: 2863-2869, doi: 10.1200/JCO.2015.61.2267

11. National Cancer Institute. Seer stat fact sheets: myeloma. http://seer.cancer.gov/statfacts/html/mulmy.html. Accessed June 19, 2015.

12. Waxman AJ, Mink PJ, Devesa SS, Anderson WF, Weiss $\mathrm{BM}$, Kristinsson SY, et al. Racial disparities in incidence and outcome in multiple myeloma: a population-based study. Blood 2010; 116: 5501-5506, doi: 10.1182/blood-2010-07298760

13. Hungria VT, Maiolino A, Martinez G, Colleoni GW, Coelho EO, Rocha L, et al. Confirmation of the utility of the International Staging System and identification of a unique pattern of disease in Brazilian patients with multiple myeloma. Haematologica 2008; 93: 791-792, doi: 10.3324/haematol.11637

14. Palumbo A, Bringhen S, Mateos MV, Larocca A, Facon T, Kumar SK, et al. Geriatric assessment predicts survival and toxicities in elderly myeloma patients: an International Myeloma Working Group report. Blood 2015; 125: 2068-2074, doi: 10.1182/blood-2014-12-615187
15. Secoli SR. [Polypharmacy: interaction and adverse reactions in the use of drugs by elderly people]. Rev Bras Enferm 2010; 63: 136-140, doi: 10.1590/S0034-71672010000100023

16. Passarelli MCG, Jacob Filho W. Reações adversas a medicamentos em idosos: como prevê-las? Einstein 2007; 5: 246-251.

17. Ferdinandi DM, Ferreira A. Alkylating agents: adverse effects and hematological complications. http://www.ciencianews. com.br/arquivos/ACET/IMAGENS/revista_virtual/hematologia/ artdamiana2.pdf. Accessed June 19, 2015.

18. Ghobrial IM, Rajkumar SV. Management of thalidomide toxicity. J Support Oncol 2003; 1: 194-205.

19. Richardson PG, Xie W, Mitsiades C, Chanan-Khan AA, Lonial $\mathrm{S}$, Hassoun $\mathrm{H}$, et al. Single-agent bortezomib in previously untreated multiple myeloma: efficacy, characterization of peripheral neuropathy, and molecular correlations with response and neuropathy. J Clin Oncol 2009; 27: 3518-35251, doi: 10.1200/JCO.2008.18.3087

20. Moreau P, Pylypenko H, Grosicki S, Karamanesht I, Leleu X, Grishunina $M$, et al. Subcutaneous versus intravenous administration of bortezomib in patients with relapsed multiple myeloma: a randomised, phase 3 , non-inferiority study. Lancet Oncol 2011; 12: 431-440, doi: 10.1016/ S1470-2045(11)70081-X

21. Arnulf B, Pylypenko H, Grosicki S, Karamanesht I, Leleu X, van de Velde $\mathrm{H}$, et al. Updated survival analysis of a randomized phase III study of subcutaneous versus intravenous bortezomib in patients with relapsed multiple myeloma. Haematologica 2012; 97: 1925-1928, doi: 10.3324/haematol.2012.067793

22. Nogales Rincon O, Huerta Madrigal A, Merino Rodriguez B, Gonzalez Asanza C, Cos Arregui E, Menchen FernandezPacheco P. Rectal bleeding and diarrhea caused by bortezomib-induced colitis. Gastroenterol Hepatol 2010; 33: 753-754, doi: 10.1016/j.gastrohep.2010.05.009

23. Mileshkin L, Stark R, Day B, Seymour JF, Zeldis JB, Prince HM. Development of neuropathy in patients with myeloma treated with thalidomide: patterns of occurrence and the role of electrophysiologic monitoring. J Clin Oncol 2006; 24: 4507-4514, doi: 10.1200/JCO.2006.05.6689 